\title{
La terapia cuádruple con bismuto durante diez días es la alterna- tiva más eficaz para erradicar el Helicobacter pylori
}

\author{
Quadruple therapy with bismuth for ten days is the most effective alternative to eradicate Helicobacter pylori
}

Clarke NP y col. JAMA Intern Med. 2015;175(7):1163-1168.

\section{Objetivos}

Comparar la eficacia y la seguridad de tres estrategias de primera línea para la erradicación de Helicobacter pylori $(\mathrm{Hp})$.

\section{Diseño, lugar y pacientes}

Ensayo multicéntrico, abierto y aleatorizado. En el contexto de un programa de rastreo para cáncer gástrico fueron reclutados mayores de 20 años con infección por Hp de nueve centros en Taiwán. Debían tener una prueba positiva de ${ }^{13} \mathrm{C}$-urea o bien, al menos dos pruebas positivas de las siguientes: test de ureasa rápido, histología, cultivo o serología.

\section{Intervención y medición de resultados principales}

De un total de 5454 pacientes invitados a participar, fueron aleatorizados 1620 (relación 1: 1:1) a alguna de las siguientes intervenciones: 1) terapia cuádruple con bismuto (Dicitrato tripotásico de bismuto $300 \mathrm{mg}$ cuatro veces al día, lansoprazol $30 \mathrm{mg}$ dos veces al día, tetraciclina 500 mg cuatro veces al día, y metronidazol $500 \mathrm{mg}$ tres veces al día) durante 10 días; 2) concomitante (lansoprazol $30 \mathrm{mg}$, amoxicilina $1 \mathrm{~g}$, claritromicina $500 \mathrm{mg}$ y metronidazol $500 \mathrm{mg}$, dos veces al día) durante diez días; 3 ) triple (lansoprazol $30 \mathrm{mg}$, amoxicilina $1 \mathrm{~g}$, claritromicina $500 \mathrm{mg}$, dos veces al día) durante 14 días.

El resultado primario fue la proporción de pacientes con erradicación exitosa de $\mathrm{Hp}$ con la primera línea de tratamiento, según intención de tratar.

\section{Resultados principales}

Los resultados principales se muestran en la tabla 1.

Podemos observar que la terapia cuádruple con bismuto fue la más efectiva, seguida por la terapia concomitante y por la triple terapia. Sin embargo, la diferencia en efectividad solo tuvo significancia estadística entre la primera y la tercera estrategia (diferencia 6,7\%; IC 95\% 2,7 a 10,7).

\section{Conclusión}

Dada la creciente resistencia a la claritromicina, la terapia cuádruple con bismuto sería preferible a la terapia triple de 14 días en el tratamiento de primera línea para Hp. La terapia concomitante administrada durante 10 días no sería óptima pero podría considerarse prolongar su tiempo de administración.

Fuentes de financiamiento: Hospital Nacional de la Universidad de Taiwán y el Ministerio de Ciencia y Tecnología de Taiwán.

Tabla 1. Proporción de erradicación exitosa de Helicobacter pylori con cada una de las tres estrategias terapéuticas comparadas.

\begin{tabular}{|c|c|c|c|c|}
\hline & Tipo de Análisis & $\begin{array}{c}\text { Cuádruple con bismuto } \\
\% \text { (IC } 95 \%) \\
\text { eventos/total pacientes }\end{array}$ & $\begin{array}{c}\text { Concomitante }{ }^{b} \\
\%(\text { (IC } 95 \%) \\
\text { eventos/total pacientes }\end{array}$ & $\begin{array}{c}\text { Triple terapiac } \\
\%(\text { IC } 95 \%) \\
\text { eventos/total pacientes }\end{array}$ \\
\hline \multirow{2}{*}{$\begin{array}{l}\text { Erradicación del } \\
\text { Helicobacter pylori }\end{array}$} & Por intención de tratar & $\begin{array}{c}90 \%(87,6 \text { a } 92,6) \\
488 / 540\end{array}$ & $\begin{array}{c}86 \%(82,7 \text { a } 88,6) \\
464 / 540\end{array}$ & $\begin{array}{c}84 \%(80,4 \text { a } 86,6) \\
452 / 540\end{array}$ \\
\hline & Por protocolo & $\begin{array}{c}96 \%(93,9 \text { a } 97,5) \\
461 / 480\end{array}$ & $\begin{array}{c}92 \%(88,9 \text { a } 93,8) \\
453 / 494\end{array}$ & $\begin{array}{c}88 \%(84,7 \text { a } 90,4) \\
446 / 508\end{array}$ \\
\hline \multicolumn{2}{|c|}{ Incidencia de efectos adversos } & $\begin{array}{c}67 \% \\
358 / 533\end{array}$ & $\begin{array}{c}58 \% \\
309 / 535\end{array}$ & $\begin{array}{c}47 \% \\
252 / 535\end{array}$ \\
\hline
\end{tabular}

a Dicitrato tripotásico de bismuto $300 \mathrm{mg}$ cuatro veces al día, lansoprazol $30 \mathrm{mg}$ dos veces al día, tetraciclina $500 \mathrm{mg}$ cuatro veces al día, y metronidazol $500 \mathrm{mg}$ tres veces al día, durante 10 días. b Lansoprazol $30 \mathrm{mg}$, amoxicilina $1 \mathrm{~g}$, claritromicina $500 \mathrm{mg}$ y metronidazol $500 \mathrm{mg}$, dos veces al día, durante diez días. c Lansoprazol $30 \mathrm{mg}$, amoxicilina $1 \mathrm{~g}$, claritromicina $500 \mathrm{mg}$, dos veces al día, durante 14 días.

\section{Comentario}

Este estudio es el primer ensayo aleatorizado de envergadura que demostró que la administración durante diez días de terapia cuádruple es más eficaz que la terapia triple durante 14 días. Por otro lado, una evaluación detallada de la susceptibilidad antibiótica de 999 pacientes que participaron en esta investigación confirmó que la terapia cuádruple durante diez días fue más eficaz que la triple durante 14 días en el subgrupo de personas con cepas resistentes a la claritromicina, y además, estos resultados permitieron estimar las eficacias de los tres regímenes en regiones con diferente prevalencia de resistencia a claritromicina.

\section{Conclusión de la comentadora}

La terapia cuádruple es una buena opción a estudiar y desarrollar en Argentina, donde la prevalencia de infección por Hp ronda $70 \%$, y donde existe una creciente incidencia de cepas con resistencia antibiótica y de cáncer gástrico.

Laura Agoff [Servicio de Gastroenterología del Hospital Italiano de Buenos Aires. laura.agoff@hospitalitaliano.org.ar]

Agoff L. La terapia cuádruple con bismuto durante diez días es la alternativa más eficaz para erradicar el Helicobacter pylori. Evid Act Pract Ambul. 2018;21(1):32. Comentado de: Jyh-Ming Liou y col. Concomitant, bismuth quadruple, and 14-day triple therapy in the first-line treatment of Helicobacter pylori: a multicentre, open-label, randomised trial. Lancet 2016;388:2355-65. PMID: 27769562

Referencias Bibliográficas

1. Dore $\mathrm{M}$ y col. Role of bismuth in improving Helicobacter pylori eradication with triple therapy. Gut $2016 ; 65: 870-88$

2. Malfertheiner P y col. Management of Helicobacter pylori infection - the Maastricht IV/Florence Consensus Report. Gut 2012; 61: 646-64

3. Fallone $\mathrm{C}$ y col. The Toronto Consensus for the treatment of Helicobacter pylori infection in adults. Gastroenterology 2016; 151: 51-69 